\title{
BILDER, DIE DIE KÖRPER BEWEGEN Neue Perspektiven auf Migration
}

von NANNA HEIDENREICH

\author{
Marie-Hélène Gutberlet, Sissy Helff, Die Kunst der \\ Migration. Aktuelle Positionen zum europäisch-afrikanischen \\ Diskurs. Material-Gestaltung - Kritik, Bielefeld \\ (Transcript) $201 \mathrm{I}$. \\ Christine Bischoff, Francesca Falk, Sylvia Kafehsy, \\ Images of Illegalized Immigration. Towards a Critical Iconology \\ of Politics, Bielefeld (Transcript) 2010 \\ FKW // Zeitschrift für Geschlechterforschung und visuelle \\ Kultur (Schwerpunkt: Visuelle Migrationen. Bild-Bewegun- \\ gen zwischen Zeiten, Medien und Kulturen) 26/I, Marburg \\ (Jonas) 20I I
}

«Der Blick entscheidet darüber, ob und wie wir Migration sehen", schrieben 2005 die Kuratorlnnen der Ausstellung des Projekts Migration ${ }^{1}$ und unternahmen damit den Versuch eines fundamentalen Perspektivwechsels. In dem transdisziplinären Projekt (2002-2006) ging es darum, Migration in einem Land, das sich über Jahrzehnte des Neologismus eines «Nichteinwanderungslandes〉 zur Selbstverständigung bediente, endlich als «zentrale Kraft gesellschaftlicher Veränderung» ${ }^{2} \mathrm{zu}$ betrachten. Mit der Einnahme der «Perspektive der Migration» ${ }^{3}$ setzte das groß angelegte Initiativprojekt der Kulturstiftung des Bundes neue Maßstäbe - und dies auch hinsichtlich der Verbindung von künstlerischem, wissenschaftlichem, kuratorischem und aktivistischem Wissen und den jeweiligen Forschungspraktiken. Die Perspektive der Migration ist dabei keineswegs deckungsgleich mit den Sichtweisen der je einzelnen Migrantinnen und Migranten, sondern impliziert, Migration als soziale Bewegung zu begreifen. Die Verbindung verschiedener Disziplinen, so das Argument des Rechtshistorikers und politischen Theoretikers Sandro Mezzadra, ist dabei in der Tat wesentlich, insofern Migration den Charakter eines fait social total hat, der es kaum erlaubt,

Migrationsbewegungen entsprechend der kanonischen Aufteilung akademischer Fächer zu untersuchen. [...] Auch noch so meisterliche wissenschaftliche Untersuchungen hinterlassen, sobald sie auf Fragen der Migrationen zu sprechen kommen, den Eindruck, dass etwas fehlt, dass es da eine Kluft gibt, die sich nur durch anspielungsreiche Worte, durch eine narrative und metaphorische Redeweise überbrücken lässt. Oder eben durch Bilder, die auf vielerlei Arten betrachtet werden können. ${ }^{4}$

Die Perspektive der Migration einzunehmen, bedeutet in diesem Sinne nicht nur, die Bilder, die mit den Bewegungen der Migration zirkulieren, die sie begleiten, die sie zeigen oder aber auch verbergen, in den Blick zu nehmen, sondern auch das Nachdenken über Migration, die theoretische Reflexion auch mithilfe von Bildern zu betreiben.

Die Verbindung von Kunst und Forschung in der Analyse von Migration ist seitdem als paradigmatisch zu sehen, wie eine Reihe von internationalen Ausstellungen und Tagungen belegt. So fanden auf Initiative von Mieke Bal und Miguel-Ángel Hernandez Navarr im Frühjahr und im Herbst 2007 die beiden Encuentro-Tagungen in Murcia und Amsterdam statt - jeweils in Verbindung mit der Ausstellung 2MOVE, in derVideokünstlerInnen Migration zum Thema ihrer Arbeiten machten und die die konstitutive Gemeinsamkeit von Bewegung, von movement, für Video und Migration als Ausgangsthese formulierte. Mieke Bal 
prägte in diesem Zusammenhang den hier wesentlichen Begriff der "migratory aesthetics», womit sie nicht nur die beiden «Bewegungsformen» Video und Migration verschalten möchte, sondern zugleich auch eine bestimmte Methode der Analyse beschreibt:

a mode that would, as anthropologist Johannes Fabian puts it, «perform s the analysis «not about, but with the people concerned. The closest I was able to come was through the medium of film, as film is a tool for making visible that which is there for everyone to see, but which remains unseen. ${ }^{5}$

Tatsächlich hat Mieke Bal neben ihrer regen Publikationstätigkeit selbst damit begonnen, Filme und Videoinstallationen zu realisieren, um jene Kultur der Bewegung, die «migratory culture», fokussieren zu können.

Diese signifikante Verbindung von Kunst und Forschung charakterisiert nun auch drei aktuelle Publikationen zum Thema Migration. Die Kunst der Migration ist Ergebnis des fünfjährigen Projekts Migration \& Media (2006-2011) unter der Federführung von Marie-Hélène Gutberlet und Sissy Helff, in dessen Rahmen sie seit 2007 Symposien, Ausstellungen, Lesungen und Filmprogramme in Frankfurt am Main und in Bamako initiiert haben. Images of illegalized Immigration hingegen ist ein klassischer Tagungsband, der die gleichnamige zweitätige Konferenz, die 2009 an der Universität Basel stattfand, nachzeichnet und in dem sich zumindest ein Teil der Beiträge mit künstlerischen Produktionen befasst. Das Heft Nr. 51 der halbjährlich erscheinenden FKW|| Zeitschrift für Geschlechterforschung und visuelle Kultur wurde in Zusammenarbeit mit der aktuellen Ausstellung des künstlerisch-wissenschaftlichen Kollektivs EUROZENTRIKA, Visual Migrations - Moving Images in Berlin erarbeitet. $^{6}$

Interessanterweise beginnen alle drei Publikationen jeweils mit einer 〈Kolumbusgeste»: mit der Feststellung, dass sie Neuland betreten. So schreibt die Herausgeberin der FKW, Kerstin Brandes, dass «[u]nter dem Titel Visuelle Migrationen ... die bisher unthematisierte Schnittstelle zwischen Migrations- und Bilddiskursen in den Blick genommen werden» soll (S.5), wohingegen die Herausgeberinnen Christine Bischoff, Francesca Falk und Sylvia Kafehsy davon ausgehen, dass sie den erstmaligen Versuch einer systematischen Untersuchung der Herstellung von Konzepten wie illegalisierter Migration durch Bilder vornehmen. Gutberlet und Helff schließlich formulieren, dass sie «[m] it der Idee, dass sich Migration als gestaltete Wahrnehmung materialisiert, im wissenschaftlichen Feld Neuland betreten.» (S.13) Diese Geste ist vermutlich jedoch nicht einfach nur als die Markierung des jeweiligen Terrains als besonders und besonders wesentlich zu verstehen, sondern spricht vielmehr für das auch vom Projekt Migration ja nicht serfundenes, sondern wesentliche Moment des Oszillierens (in) der Migration, das zu steten Verschiebungen und ständigen Neuaufbrüchen in der Thematisierung führt. Was die drei Bände jedoch in der Tat als neu kennzeichnet, ist, dass hier Migration nun vor allen Dingen unter bild- und kunstwissenschaftlichen Gesichtspunkten fokussiert wird. Die damit einhergehende Vernachlässigung eines gesellschaftswissenschaftlichen Referenzrahmens birgt jedoch die Gefahr, erneut disziplinär zu verengen.

\section{Migrierende Bilder}

Bilder sind zunächst konstitutiv für die Herstellung verschiedener Migrationsregimes. Insbesondere gilt dies für die illegalisierte Migration, die gerne auch als «unsichtban imaginiert wird. So hat die Filmemacherin und Künstlerin Brigitta Kuster bereits 2007 die Frage aufgeworfen, inwiefern die zirkulierenden Bilder und Erzählungen «über Migration» unerlässliche Ressourcen sind, um «Migration» zu verstehen. Bilder, so ihr Argument, «formatieren» Migration. ${ }^{7}$ Mit Blick auf zeitgenössische Dokumentarfilme, die die klandestine Migration in den Blick nehmen und auf jeweils unterschiedliche Weise den Versuch einer kritischen Sichtbarkeitspolitik unternommen haben, resümiert Kuster, dass jeder Versuch, eine alternative Form der Darstellung zu schaffen, sich dabei in dem Bildarchiv des Grenzregimes zurechtfinden muss, in jener «visuellen Ökonomie

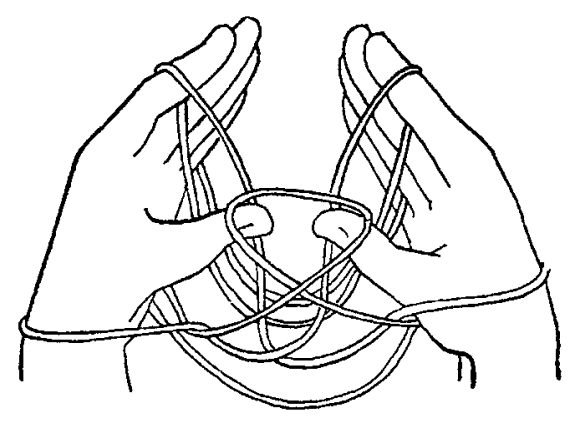


der Kriminalisierung, Regulation und Kontrolle» mit ihrer «Despotie der Identifizierung». ${ }^{8}$ Entsprechend widmet sich der Band Images of Illegalized Immigration diesem Bildarchiv, einer genauen Lektüre seiner Bestände sowie künstlerischen Infragestellungen hegemonialer Ansprüche. Den Band eröffnet ein Beitrag von W. J.T. Mitchell, dessen Betrachtung «migrierender Bilden bereits 2004 den Titel einer Tagung am Berliner Haus der Kulturen der Welt gestiftet hatte. Damals stellte er die Frage, was es bedeuten würden, von Bildern wie von Migrantlnnen zu sprechen, und schlug vor, die Zirkulation von Bildern als Migration zu beschreiben. ${ }^{9}$

Hier nun konkretisiert Mitchell seine These anhand eines spezifischen Szenarios der faktischen Migration, dem des Checkpoints, sowohl in seinen realen als auch in seinen fiktiven beziehungsweise fiktionalisierten Varianten. Er eröffnet seinen Beitrag mit der Feststellung, dass die Betrachtung der Bilder illegalisierter Migration drei Felder zu verbinden habe: das des Rechts, jenes der Migration selbst sowie schließlich das der Ikonologie. Diese verbindet er mittels des Rawls'schen «Schleier des Nichtwissens» und entwickelt daraus eine Utopie, die er mit der Hoffnung auf die gestaltende Kraft der Bilder verknüpft: «We will continue to need the veil of ignorance in order to secure any notion of legality with respect to migration; we will need to rend the veil, and project new images on it in order to have any hope of justice.» (S. 30). Mitchells luzide Verknüpfung von Rechts- und Bildtheorie findet leider keine Resonanz in den weiteren Beiträgen des Bands; es findet sich lediglich eine kurze Erwähnung des von Agamben theoretisierten «nackten Lebens» in Pamela C. Scorzins Beitrag Voice-Over Image (S.108), der als einziger nicht nur Bilder thematisiert, sondern sich den Möglichkeiten des Sounds für eine Sichtbarmachung der unsichtbaren Migration widmet. Ein Großteil der Beiträge bleibt zudem schlicht einem repräsentationskritischen Ansatz verhaftet, mit dem die meist in den Massenmedien verbreiteten Bilder, etwa von Flüchtlingen (Almut Rembges) oder Opfern von 〈Frauenhandel〉 (Sylvia Kafehsy), als Stereotype kritisiert werden. Dies kulminiert im Beitrag der Mitherausgeberin Christine Bischoff in dem Kurzschluss: «Give to a group a bad image and it will correspond to it.» (S.48) Bischoff betrachtet die Schweizer Medienhysterie um die Auto rasenden «Speed-Junkies» aus dem «Balkan〉 (die natürlich oft genug Schweizer Staatsbürger sind) und analysiert damit Illegalisierung unter den Stichworten Kriminalisierung und Devianz. Ähnlich geht auch Jan-Henrik Friedrichs vor, der sich in Milieus of
Illegality den Repräsentationen der Gastarbeiter in Der Spiegel aus den Jahren 1973-1980 widmet (Stichwort: 〈Ghetto〉) und damit einen wichtigen Beitrag zur Historisierung von Illegalisierung leistet.

Generell leidet der Band vor allen Dingen an dem teilweise holprigen Englisch sowie an der die Vortragsmanuskriptlänge nicht übersteigenden Kürze der Beiträge. So bleiben einige vielversprechende Untersuchungen wie der Versuch, eine Ikonologie der illegalisierten Immigration vorzulegen (Francesca Falk), nur kursorisch. Gleiches gilt für den ebenfalls positiv auffallenden Beitrag von Michael Andreas, der sich einer Vielzahl von theoretischen Impulsen bedient und u. a. die Autonomie der Migration (Yann Moulier-Boutang), das (Tier-)Werden (Gilles Deleuze), Homi K. Bhabhas Konzept der Mimikry und Michael Taussigs Mimesis in Verbindung mit der Serie Undocumented Interventions des Künstlers Julio Morales diskutiert.

Andreas bezieht sich auch explizit auf Bals migratory aesthetics, wodurch die Verbindung nicht nur von Kunst und Forschung, sondern auch von Kunst und Aktivismus, die im Projekt Migration noch explizit angelegt war, kurz aufscheint: «what was artistic becomes activist» resümiert er als Resultat der kuratorischen Anordnung (S. 65). Diese Engführung von kuratorischer Praxis und Aktivismus näher zu erläutern bleibt er jedoch schuldig.

\section{Kunst der Migration}

Dass und wie Kunst und politische Anliegen in einem Austauschverhältnis stehen, zeigt anschaulich der wesentlich umfangreichere Band Die Kunst der Migration. Er versammelt unterschiedliche Textformen, wissenschaftliche Beiträge, Autobiografisches, künstlerische Statements, Gedichte und Arbeitsnotizen, mit denen der Versuch unternommen wird, eine "Ästhetik der Migration» auszumachen und zu formulieren (S.11). Mit Ästhetik ist hier sowohl die Wahrnehmung von Migration als auch die Form der Wahrnehmung gemeint, in der sich Migration materialisiert. Die Herausgeberinnen entfernen sich in diesem Sinne explizit von einer reinen «Kritik der Medialisierung von Migrationstopoi» (ebd.) und stellen die Frage nach der Sicht der Akteurlnnen, nicht im Sinne einer BetroffenenÄsthetik, sondern vielmehr verstanden (wenn auch nicht so benannt) als «Perspektive der Migration». Dazu gehört auch, dass die durch die transnationalen Bewegungen der Migration entstehenden neuen Räume «des Denkens und Gestaltens» eine Neuerfindung von Methoden jenseits herkömmlicherDisziplinen erforderlich gemachthaben(S.12). 

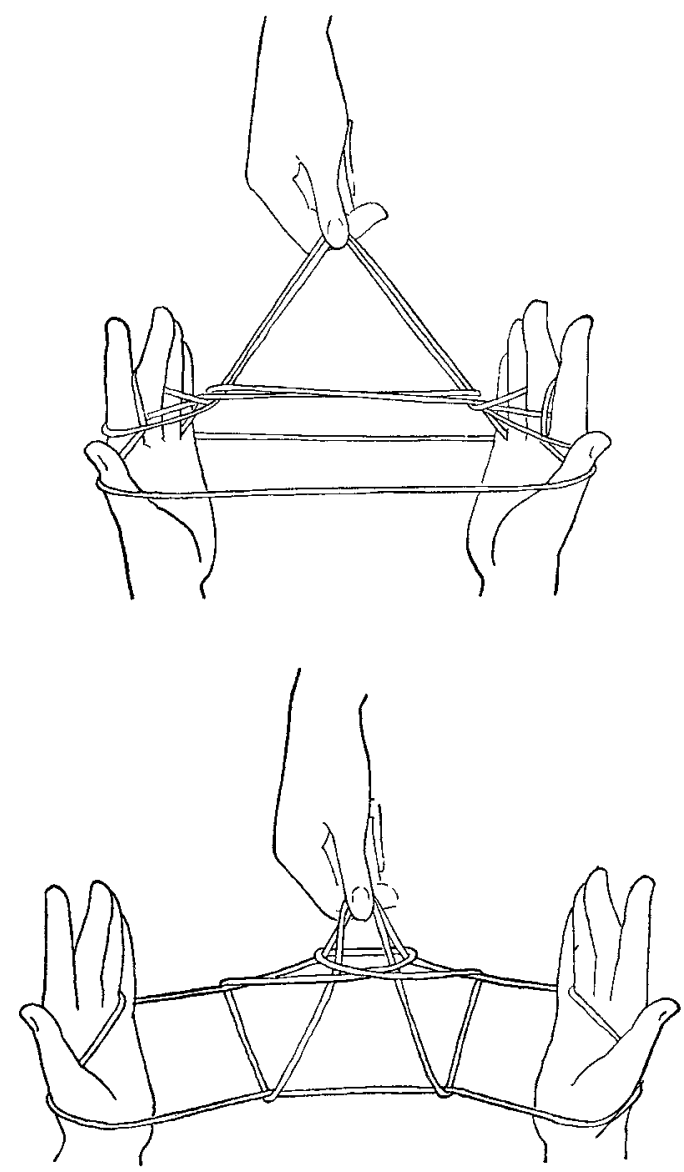

Das Buch ist in drei Abschnitte unterteilt, Filmische Parallaxen, Grenzen in Visual Culture und Mobile Narrative, wobei der letzte, der sich literarischen Praktiken widmet, hier vernachlässigt werden soll. In den Filmischen Parallaxen stehen neben einer kritischen Betrachtung der unterschiedlichen Produktions- und Rezeptionsräume Deutschland und Frankreich durch den Filmemacher Idrissou MoraKapi Beiträge, die dichte Analysen einzelner Filme liefern, wobei der Blick auf solche Produktionen gelenkt wird, die Bilder «bekommen anstatt sie erbeuten zu wollen» (MarieHélène Gutberlet, S. 55), die sich «dem exotischen und miserabilistischen Blick des Westens auf den afrikanischen Kontinent» widersetzen (Julien Enoka Ayemba, S. 61) und inmitten komplexer intertextueller Bezüge Raum schaffen für individuelle Geschichten, die von der Last der Stellvertreterfunktion befreit werden (Dirk Naguschewski, S. 90).
Besonders eindrückliche Artikel finden sich jedoch im mittleren Teil des Bandes, der sich den Grenzen in Visual Culture widmet, wo das von den Herausgeberinnen anvisierte Geflecht eines Austauschs im Sinne der Neuperspektivierung von Migration unter anderem in den miteinander korrespondierenden Beiträgen von Brigitta Kuster, Moise Merlin Mabouna und Marie-Hélène Gutberlet erfolgreich zum Tragen kommt. Kuster und Mabouna reflektieren die Zusammenarbeit am ersten gemeinsamen Videoprojekt Rien ne vaut que la vie mais la vie même ne vaut rien (2002/2003), über deren Installation und Präsentation im Rahmen von Migration \& Medien wiederum Marie-Hélène Gutberlet nachdenkt, und dies wird schließlich durch einen Text von Brigitta Kuster über den Entschleunigungszustand ¿Camp〉 ergänzt.

\section{Migration der Form}

Ein weiterer Stichwortgeber für die aktuellen Diskussionen ist die Rede von der «Migration der Form(en)», dem Leitthema der Documenta 12 (2007), das auch Gutberlet und Helff in der Einleitung zitieren, um auf die Zirkulation der Bilder im globalen Kunstkontext zu referieren. Dies wird vor allen Dingen in den Beiträgen von Bärbel Küster, die sich den Austauschprozessen in der Kunst zwischen Afrika und Europa widmet, und von Ulf Vierke, der die künstlerische Praxis afrodeutscher und afrikanischer KünstlerInnen zusammen mit Rezeptionsmustern entlang der gängigen «Bilder von Afrika〉 betrachtet, aufgegriffen. Wirklich neue Felder werden jedoch erst in den Artikeln erschlossen, die sich digitalen Medien widmen. Hier ist Sissy Helffs Auseinandersetzung mit Weblogs im Spannungsfeld von Authentizitätsdiskursen und Rassismus zu nennen. Besonderes Augenmerk verdient Soenke Zehles Beitrag Spiel ohne Grenzen zu Serious Games zwischen dokumentarischer Übersetzung und Kartographien des Politischen, in dem die aus den Debatten um das Verhältnis von Kino bzw. Kunst und Politik vertraute Frage des Verhältnisses von Ästhetik und (oder) Politik völlig neu verhandelt wird und zugleich die Spieltheorien mit den «ethischen und ästhetischen Fragen der Grenzen einer Politik der Repräsentation» konfrontiert werden (S. 113). Können Serious Games als Werkzeuge des Aktivismus dienen, als Medium politischer Reflexion oder gar zur Hervorbringung politischen Engagements? Zehle argumentiert überzeugend, dass die Spiele vor allem heuristische Instrumente seien und verortet deren Potenzial damit in der «prozeduralen Rekonfiguration des Politischen» (S. 131). 

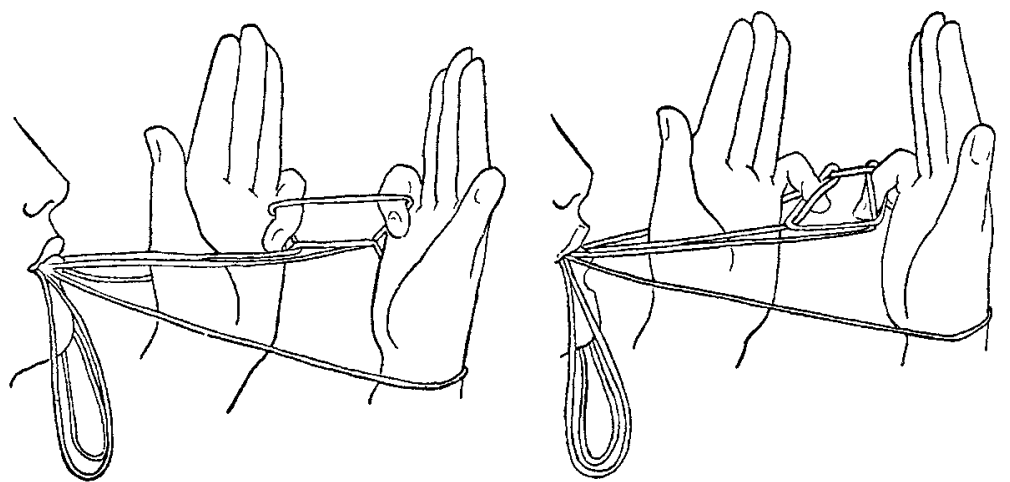

Die FKW bezieht sich auch auf Mitchells bildtheoretische Analogisierung von migrierenden Bildern und MigrantInnen und fokussiert verschiedene Formen zirkulierender und migrierender Bilder, wobei diese nicht notwendigerweise solche der Migration selbst sind, sondern auch Bilder rassistischer und kolonialer Imaginationen.

Silke Wenk und Rebecca Krebs richten in ihrem Beitrag die Aufmerksamkeit aufFotografien aus den Anfängen der Gastarbeiterära. Die Deutung von Bildern, so die Autorinnen, ist nicht nur eine Sache der Bilder selbst, sondern wird auch von denjenigen bestimmt, die über die «medialen Apparate» verfügen (S.15). Zentralen Stellenwert nimmt in der FKW-Ausgabe das Bild des Bootes ein, als Metapher der Migration («das Boot ist voll») bei Wenk und Krebs, aber auch als Trope kritischer künstlerischer Praxis in Melanie Ulz' Beitrag, in dem Beispiele aus der internationalen Gegenwartskunst herangezogen werden, die das Schiff je unterschiedlich einsetzen, um damit in den «(visuellen) Diskurs um Migration, Integration und Abgrenzung zu intervenieren.» (S. 31) Astrid Kussers Beitrag, der sich wie Ulz auf das Konzept des Black Atlantic bezieht und sich mit Bildpostkarten um 1900 und den darin enthaltenen Inszenierungen einer Schwarz-Weiß-Dynamik befasst, spitzt die Mitchellsche These am prägnantesten auf eine explizite Verknüpfung von Bild- und Migrationstheorie zu: «Bilder waren das Ergebnis von Migration, Ausdruck einer Abweichung vorgesehener Zirkulationsrouten, sichtbares Dokument eines gesellschaftlichen Imaginären, das nicht nur kolonialen Kartografien folgte, sondern in der Tradition des Black Atlantic stand.» (S. 50) Sie entdeckt in den Postkarten ein visuelles Archiv, das die Geschichte eines auch mittels Bildern ausgehandelten Konfliktes erzählt, Bilder, «die einander widersprachen, aufeinander antworteten oder voreinander flohen.» (Ebd.)

Zum Schluss nochmals zurück zu den Anfängen: Die Ausstellung im Kölnischen Kunstverein 2005 markierte auch ein 〈Jubiläum〉 bundesdeutscher Migrationsgeschichte, den 50. Jahrestag der Unterzeichnung des italienischdeutschen Anwerbeabkommens. Dieses Jahr nun wird in vielfältigen Veranstaltungen des 1961 unterzeichneten Anwerbeabkommens mit der Türkei gedacht, unter anderem in Ausstellungen. Es bleibt abzuwarten, wie der Versuch einer Verknüpfung von Bildern und Migration im Sinne einer Neuverortung jenseits von Quantifizierung und Populismus, zu dem auch die genannten Publikationen beitragen wollen, sich tatsächlich etablieren konnte.

1 Aytaç Eryılmaz u. a., Vorwort, in: Kölnischer Kunstverein u. a. (Hg.), Projekt Migration, Ausstellungskatalog, Köln (DuMont) 2005, 16-21, hier 16. Die Ausstellung des Projekts Migration fand vom 30.9.05 bis 15.6 .06 im Kölnischen Kunstverein und an zwei weiteren Orten statt. Das Kuratorium des Gesamtprojekts setzte sich zusammen aus: Aytaç Eryılmaz, Marion von Osten, Martin Rapp, Kathrin Romberg und Regina Römhild.

2 Ebd.

3 Ebd.

4 Sandro Mezzadra, Lo sguardo dell'autonomía / Der Blick der Autonomie, in: Kölnischer Kunstverein u. a. (Hg.), Projekt Migration, Köln (DuMont) 2005, 794-795, hier 794 .
5 Mieke Bal, Migratory Aesthetics: Double Movement, in: Exit, 4/8, 2008/2009, http://www.exitmedia. net/prueba/eng/articulo.php?id $=266$, gesehen am 07.07.2011.

6 Die Ausstellung fand im Juli 2011 bei Leap in Berlin statt, siehe dazu http://eurozentrik.net, gesehen am 07.07.2011.

7 Brigitta Kuster, Die Grenze filmen, in: Transit Migration Forschungsgruppe (Hg.), Turbulente Ränder. Neue Perspektiven auf Migration an den Grenzen Europas, Bielefeld (Transcript), 187-202, hier 187 .

8 Ebd., S. 188.

9 W.J.T. Mitchell, Migrating Images. Totemism, Fetishism, Idolatry, in: Petra Stegemann, Peter C. Steel (Hg.), Migrating Images, Berlin (Haus der Kulturen der Welt) 2004, 14-24. 